\title{
Involvement of TP53 and TP16 expression in human papillomavirus-associated non-small cell lung cancer
}

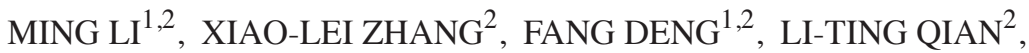 \\ SHUI-PING MENG ${ }^{3}$, WU-LIN SHAN ${ }^{2}$ and BAO-LONG WANG ${ }^{1}$ \\ ${ }^{1}$ Department of Clinical Laboratory, Affiliated Provincial Hospital of Anhui Medical University, \\ Hefei, Anhui 230001; Departments of ${ }^{2}$ Clinical Laboratory and ${ }^{3}$ Respiratory Oncology, \\ Anhui Provincial Cancer Hospital, Hefei, Anhui 230031, P.R. China
}

Received February 3, 2015; Accepted July 26, 2016

DOI: $10.3892 / 01.2016 .5087$

\begin{abstract}
Human papilloma virus (HPV) infection has previously been reported to be associated with TP53 and TP16 expression in Japanese and Taiwanese patients with lung cancer, but data for advanced non-small cell lung cancer (NSCLC) patients is limited. The present study examined the association between HPV infection and TP53 and TP16 expression in Chinese patients with advanced NSCLC. HPV DNA was detected in 20 out of 83 (24\%) lung tumors, and was observed more frequently in non-smokers, patients with lymph node metastasis, and patients with poorly differentiated tumors $(\mathrm{P}=0.048, \mathrm{P}=0.044$ and $\mathrm{P}=0.024$, respectively $)$. Thirteen $(65 \%)$ out of $20 \mathrm{HPV}$-infected tumors were positive for TP53 expression while eight (40\%) were positive for TP16 expression. Multivariate analysis revealed that poor differentiation alone $(\mathrm{OR}=0.163)$ was an independent predictive factor of HPV infection in NSCLC. TP16-positive patients had a significantly longer survival time when compared with TP16-negative patients $(\mathrm{P}<0.001, \log$-rank test $)$, a trend a not observed for TP53. Our results suggest that TP53 and TP16 protein expression is not associated with the expression of HPV DNA, but that TP16 expression may be an independent prognostic factor of long survival in advanced NSCLC.
\end{abstract}

\section{Introduction}

Non-small cell lung cancer (NSCLC) accounts for the majority of all lung cancers, and represents the leading cause of cancer-related mortality worldwide. The pathogenesis of NCSLC is complex and is believed to occur as a result of

Correspondence to: Professor Bao-Long Wang, Department of Clinical Laboratory, Affiliated Provincial Hospital of Anhui Medical University, 17 Lujiang Road, Hefei, Anhui 230001, P.R. China

E-mail: 18919685630@163.com

Key words: human papillomavirus, TP16, TP53, non-small cell lung cancer, survival interactions between environmental and genetic factors $(1,2)$. Cigarette smoking, environmental pollution, and radiation have been identified as the main risk factors of NSCLC. However, not every type of lung carcinoma is associated with these factors, and additionally, oncogenic types of HPV have also been proposed as potential causes of $\operatorname{NSCLC~}(3,4)$.

HPVs are double-stranded, non-enveloped DNA viruses. Recent studies suggest that HPV infection is an important cause of cervical cancer $(5,6)$. HPVs are commonly divided into high-risk and low-risk types, with the high-risk type HPV 16 representing the predominant type associated with cervical cancer (7). A number of research groups have reported a high detection rate of HPV in lung cancer tissues, particularly HPV 16 and 18, suggesting a role for HPV in promoting this disease (8-10). Although HPV has been hypothesized to be a possible contributory agent for lung cancer, its role in the pathology of this disease remains controversial. This controversy relates to regional and organizational differences in HPV infection, as well as its unknown pathogenesis in lung cancer (11-13). Identification of diagnostic markers for HPV-associated NSCLC is necessary both to clarify the relationship between HPV infection and NSCLC pathogenesis and to improve therapeutic strategies.

TP53 is an important regulator of the cell cycle, and alterations in the TP53 gene are frequently observed in invasive tumors (14). A number of previous studies have demonstrated that the HPV E6 protein binds TP53 and triggers its degradation through the ubiquitin pathway, promoting proliferation and inhibition of apoptosis $(15,16)$. In addition, a significant correlation between HPV infection and TP16 expression has been observed in a variety of HPV-associated tumor specimens (17-19). Overexpression of TP16, a cyclin-dependent kinase inhibitor, can prevent the phosphorylation of the retinoblastoma protein, an important cellular tumor suppressor, resulting in its activation and consequent inhibition of cell cycle progression (20). While there is accumulating evidence that the HPV 16/18 E6 oncoprotein is indeed expressed in lung tumors and is involved in TP53 and TP16 signaling, the correlation between HPV infection and the expression of these proteins remains controversial. Consequently the expression of TP53 and TP16 in relation to that of the E6 oncoprotein in NSCLC requires further clarification. 
Although heterogeneity in the expression of TP53 and TP16 has been demonstrated to correlate with similarly heterogeneous clinical outcomes in other tumor types, these relationships have been more difficult to evaluate for NSCLC. Evaluation of TP53 and TP16 mutation by standard techniques requires tissue quantities that are often only available from resected tumors $(21,22)$. For this reason, the majority of studies of TP53 and TP16 status in NSCLC have been performed during the early stages of disease. The small numbers of patients included in some of these studies, differences in follow-up, and the various criteria used to classify TP53 and TP16 mutation have led to contradictory results. In comparison with TP53, the role of TP16 in HPV-related NSCLC has received little attention.

In the present study, we have analyzed TP53 and TP16 expression in a training cohort of 83 patients with advanced NSCLC, and evaluated the relationship between the expression of these proteins and various clinical parameters. The findings were evaluated in an independent cohort of 20 HPV-positive patients.

\section{Materials and methods}

Clinical specimens. The present study was a retrospective analysis of a total of 83 patients with advanced NSCLC, grouped according to HPV status. All patients were diagnosed with stage III-IV disease at the Anhui Provincial Hospital between August 2011-August 2013. Patient samples included 17 surgical specimens, 12 lung biopsy specimens, 15 bronchoscopic biopsy specimens, 24 pleural effusion specimens, 13 lymph node biopsy specimens and two bone biopsy specimens. In all cases, cancer-containing tissues were obtained at the time of diagnosis of metastatic disease. Written informed consent was provided by all patients, and approval was obtained from the Institutional Review Board of Anhui Provincial Hospital. Clinical characteristics including age, sex, smoking history, histology type, histological differentiation, lymph node metastasis, distant metastasis, and expression of TP53 and TP16 were collected for subsequent analyses.

Processing of tumor samples and DNA extraction. All samples used in the present study were formalin-fixed, paraffin-embedded tumor tissues that had previously been evaluated by an expert pathologist. To deparaffinize and extract DNA from tissue sections, the QIAamp DNA FFPE Tissue Kit (cat. no 56404, QIAGEN, Hilden, Germany) was used and samples were processed according to the manufacturer's recommendations. Briefly, 5-6 formalin-fixed, paraffin-embedded $8-\mu \mathrm{m}$ sections were soaked in xylene and vortexed vigorously. The extracted tissue was then pelleted by centrifugation $(20,000 \mathrm{x}$ g) and the DNA subsequently purified following the kit's protocol. All DNA samples were quantified by spectrophotometric absorbance at $260 \mathrm{~nm}$ and aliquots of equal concentration were then prepared for all samples.

Detection and typing of HPV-DNA. HPV testing of NSCLC samples was performed by PCR amplification of a fragment of the HPV L1 gene, and the subsequent screening of PCR products using a Tellgenplex ${ }^{\mathrm{TM}}$ HPV DNA Test kit (Tellgen, Shanghai, China) in combination with the Luminex technique, which allowed the detection of 26 HPV genotypes including 19 high-risk types (HPV 16, 18, 26, 31, 33, 35, 39, $45,51,52,53,55,56,58,59,66,68,82$, and 83) and seven low-risk types (HPV 6, 11, 40, 42, 44, 61, and 73). The experiments were performed using a Bio-Plex 200 system (Bio-Rad, Hercules, CA, USA) according to the manufacturer's instructions. Briefly, $2 \mu \mathrm{g}$ of the extracted DNA was used as a template for PCR, and the reaction was performed as follows. A denaturing step of $95^{\circ} \mathrm{C}$ for $30 \mathrm{sec}$, followed by an annealing step of $58^{\circ} \mathrm{C}$ for $30 \mathrm{sec}$, and then an extension step of $72^{\circ} \mathrm{C}$ for $30 \mathrm{sec}$ were performed for the first five cycles, and then an additional 35 cycles were performed as described except that the annealing temperature was reduced to $55^{\circ} \mathrm{C}$. The PCR products were then subjected to fast hybridization and the data were analyzed with the software supplied by the manufacturer (23).

Qualitative analysis of TP53 and TP16 protein expression. Paraffin-embedded tumor tissue sections were deparaffinized and rehydrated through a series of graded alcohol washes. Microwave-based antigen retrieval was conducted using $10 \mathrm{mM}$ citric acid buffer ( $\mathrm{pH}$ 6.0). Sections were blocked in normal rabbit serum (Dako North America, Inc., Carpinteria, CA, USA) containing $10 \%$ (v/v) stock avidin solution (Themo Fisher Scientific, Inc., Waltham, MA, USA) for $1 \mathrm{~h}$ followed by a 12 -h incubation at $4^{\circ} \mathrm{C}$ with mouse monoclonal antibodies against human TP53 or TP16 (Cell Signaling, Beverly, MA, USA). Sections were then incubated with biotinylated anti-mouse antibody (for monoclonal antibodies) for $1 \mathrm{~h}$ and finally treated with the chromogenic agent diaminobenzidine (Dako) for $5 \mathrm{~min}$. Sections were counterstained with Mayer's hematoxylin. Samples were assessed by light microscopy, and TP16 and TP53 positivity was evaluated according to the intensity and expected pattern of cellular staining.

Statistical analysis. All statistical analyses were performed using SPSS software version 16.0 (SPSS Inc., Chicago, USA). Chi-square tests were used to examine associations between HPV status in tumors and clinical characteristics (age, sex, smoking history, histology type, differentiation, lymph node metastasis, distant metastasis, and TP53 and TP16 expression). Progression-free survival (PFS) was defined as the time from diagnosis to recurrence or the date of censorship (the last date of follow-up). We analyzed the combined effects of HPV infection and over-expression of TP53 and TP16 on PFS. Survival curves were estimated using the Kaplan-Meier method and differences in survival distributions were evaluated using a log-rank test. To evaluate mortality risk, the hazard ratio (HR) and the corresponding confidence interval (CI) were estimated using Cox proportional hazards models to identify potential prognostic factors. All statistical tests were two-sided and $\mathrm{P}<0.05$ was considered to indicate a statistically significant difference.

\section{Results}

TP53 and TP16 expression in NSCLC. To evaluate the expression of TP53 and TP16 in NSCLC tissues, qualitative, 
Table I. Clinical characteristics of patients, grouped according to HPV status.

\begin{tabular}{|c|c|c|c|}
\hline \multirow[b]{2}{*}{ Characteristic } & \multicolumn{2}{|c|}{ HPV } & \multirow[b]{2}{*}{ P-value } \\
\hline & Positive & Negative & \\
\hline Age & & & 0.965 \\
\hline$\leq$ Median age & $11(13.3)$ & $35(42.2)$ & \\
\hline$>$ Median age & $9(10.8)$ & $28(33.7)$ & \\
\hline Sex & & & 0.344 \\
\hline Female & $9(10.8)$ & $21(25.3)$ & \\
\hline Male & $11(13.3)$ & $42(50.6)$ & \\
\hline Differentiation & & & 0.024 \\
\hline Well & $2(2.4)$ & $23(27.7)$ & \\
\hline Poor & $18(21.7)$ & $40(48.2)$ & \\
\hline Smoking status & & & 0.048 \\
\hline Never smokers & $13(15.7)$ & $25(30.1)$ & \\
\hline Current and former smokers & $7(8.4)$ & $38(45.8)$ & \\
\hline Histology & & & 0.224 \\
\hline Squamous & $17(20.5)$ & $45(54.2)$ & \\
\hline Adenocarcinoma & $3(3.6)$ & $18(21.7)$ & \\
\hline Lymph node metastasis & & & 0.044 \\
\hline Yes & $9(10.8)$ & $44(53)$ & \\
\hline No & $11(13.3)$ & $19(22.9)$ & \\
\hline Distant metastasis & & & 0.282 \\
\hline Yes & $9(10.8)$ & 37 (44.6) & \\
\hline No & $11(13.3)$ & $26(31.3)$ & \\
\hline Expression of TP16 & & & 0.496 \\
\hline Negative & $12(14.5)$ & $43(51.8)$ & \\
\hline Positive & $8(9.6)$ & $20(24.1)$ & \\
\hline Expression of TP53 & & & 0.456 \\
\hline Negative & $7(8.4)$ & $28(33.7)$ & \\
\hline Positive & $13(15.7)$ & $35(42.2)$ & \\
\hline
\end{tabular}

HPV, human papilloma virus; TP16, tumor suppressor protein p16; TP53, tumor suppressor protein p53.

intensity-based analysis of tissue staining was performed. Histological samples were assigned one of four possible scores, as follows: No detectable expression, detectable nuclear staining, easily visible nuclear staining, and strong staining. Samples that were scored as "no detectable expression' were defined as negative; samples scored as 'strong staining' were defined as positive for TP53 or TP16 expression. Representative examples of NSCLC patient tissue samples stained for TP53 or TP16 that scored as either positive or negative are shown in Fig. 1.

HPV status and TP53 and TP16 expression scores. Table I summarizes the clinical characteristics of the 83 patients with advanced NSCLC included in this study. Patients were assigned to one of two groups according to their HPV status, as determined by PCR-based HPV DNA screening of tissue specimens. Of the 83 specimens examined, 20 were HPV-positive and 63 were HPV-negative. HPV 16 was the most common subtype detected, although HPV 18, 33, 58,
6 and 11 were also detected. HPV infection was significantly associated with poor differentiation $(\mathrm{P}=0.024)$, never smoking $(\mathrm{P}=0.048)$, and lymph node metastasis $(\mathrm{P}=0.044)$. A total of $28(33.7 \%)$ patients were found to be TP16-positive and $48(57.8 \%)$ were found to be TP53-positive. However, the presence of HPV DNA did not significantly correlate with positive TP53 or TP16 expression status (Table I). Multivariate analysis revealed that poor differentiation $(\mathrm{OR}=0.163)$ was an independent predictive factor of HPV infection in advanced NSCLC (Table II). This phenomenon was not found in the equation of logistic regression analysis for HPV infection (Table III).

Effects of HPV status and positive TP16 expression on progression-free survival (PFS). To examine the clinical significance of HPV infection as well as TP16 and TP53 expression status in advanced NSCLC, we evaluated their impact on PFS. Survival curves were generated using Kaplan-Meier estimates (Fig. 2). HPV-positive patients 
Table II. Multivariate analysis of predictive factors for HPV infection.

\begin{tabular}{|c|c|c|c|}
\hline Characteristic & OR & $95 \% \mathrm{CI}$ & P-value \\
\hline Age $(\leq$ median age vs. $>$ median age $)$ & 1.129 & $0.337-3.781$ & 0.844 \\
\hline Sex (female vs. male) & 0.639 & $0.135-3.029$ & 0.572 \\
\hline Differentiation (well vs. poor) & 0.163 & $0.030-0.896$ & 0.037 \\
\hline Smoking status (never smokers vs. current and former smokers) & 0.348 & $0.075-1.610$ & 0.177 \\
\hline Histology (squamous vs. adenocarcinoma) & 0.282 & $0.050-1.600$ & 0.153 \\
\hline Lymph node metastasis (yes vs. no) & 0.364 & $0.099-1.347$ & 0.130 \\
\hline Distant metastasis (yes vs. no) & 0.638 & $0.168-2.416$ & 0.508 \\
\hline Expression of P16 (low vs. high) & 1.087 & $0.324-3.653$ & 0.893 \\
\hline Expression of P53 (low vs. high) & 2.649 & $0.749-9.367$ & 0.131 \\
\hline
\end{tabular}

HPV, human papilloma virus; OR, odds ratio; CI, confidence interval.
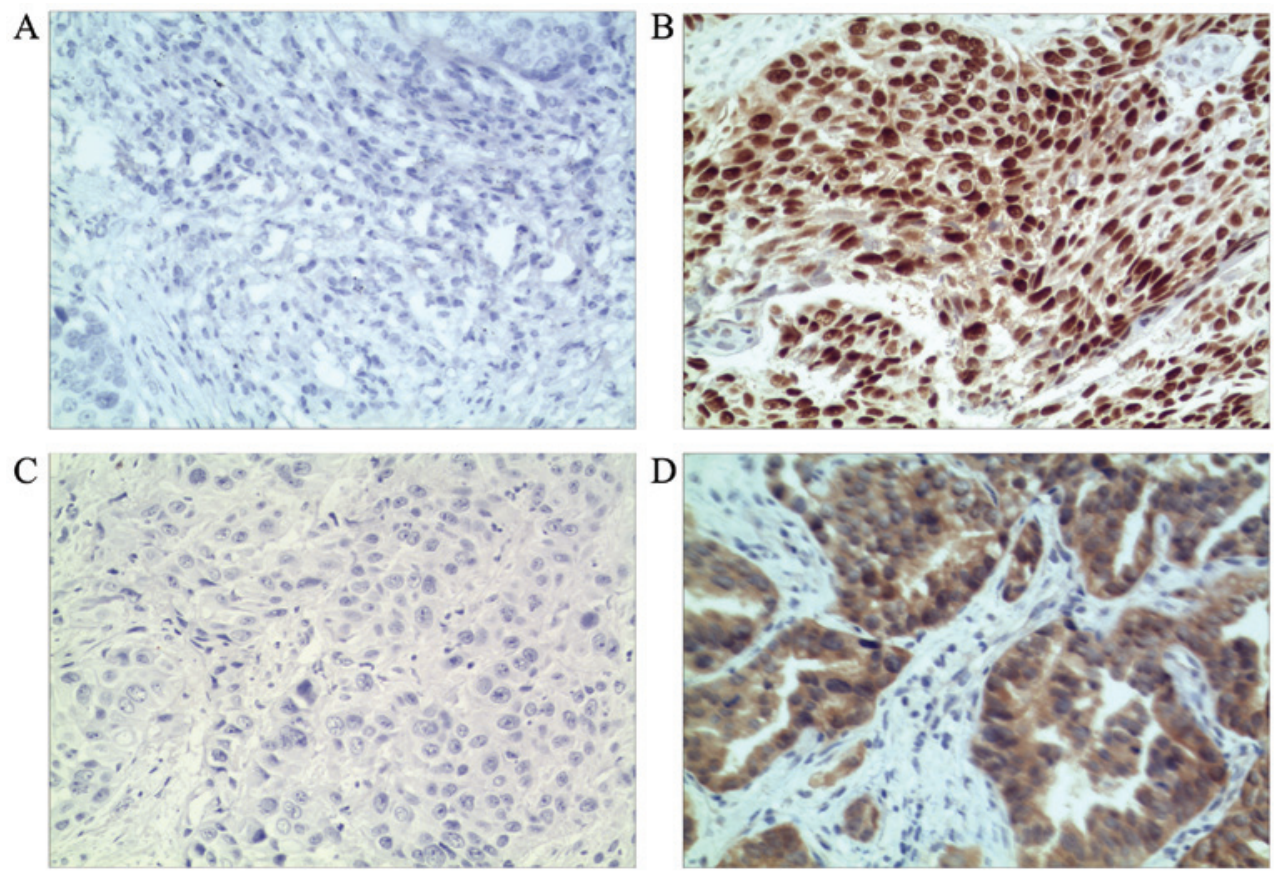

Figure 1. Expression of TP53 and TP16 in NSCLC. Immunohistochemistry was performed to qualitatively analyze the expression of TP53 and TP16 in tumor tissues. Micrographs show examples of (A) TP53-negative tissue staining, (B) TP53-positive tissue staining, (C) TP16-negative tissue staining, and (D) TP16-positive tissue staining. Images were acquired at x400 magnification.

had a median PFS of 12 months compared with 8 months for HPV-negative patients. However, this difference was not statistically significant (HR, 0.68; 95\% CI, 0.409-1.161; $\mathrm{P}=0.162$ ).

As shown in Table IV, TP53 expression status had no significant effect on patient survival (HR, 1.102; 95\% CI 0.698-1.738; $\mathrm{P}=0.678)$. However, when we analyzed the impact of TP16 expression status on survival we found that TP16-positive patients had a median PFS of 11 months compared with 8 months for TP16-negative patients, and this difference was statistically significant (HR, 0.562; 95\% CI, 0.341-0.924; $\mathrm{P}=0.023$ ).

In addition, our data confirmed that patients who never smoked had a longer PFS than smokers, while patients with adenocarcinoma had a longer PFS than those with squamous cell carcinoma. Multivariate analysis also demonstrated that histology type and lymph node metastasis were significant determinants of PFS.

\section{Discussion}

The present study demonstrated that, of the 83 Chinese patients with advanced NSCLC, the overall frequency of HPV detection was 24\%, and that HPV 16 was the mostly commonly detected type. Our data indicate that HPV infections were associated with non-smokers, patients with lymph node metastasis and patients with poorly differentiated tumors. In our multiple biomarker assessment (namely, HPV, TP53 and TP16 status) we identified that TP16-positive patients exhibited longer survival times when compared with 
Table III. Variables in the equation of logistic regression analysis for HPV infection.

\begin{tabular}{lccccc}
\hline Variable & $\beta$ & Sample mean & Wald value & RR & 95\% CI \\
\hline HPV infection & -0.382 & 0.282 & 1.841 & 0.682 & $0.393-1.185$ \\
TP16 & -0.514 & 0.259 & 3.946 & 0.598 & $0.772-2.006$ \\
TP53 & 0.219 & 0.244 & 0.809 & 1.245 & $0.360-0.993$ \\
Differentiation & -0.220 & 0.265 & 0.691 & 0.802 & $0.477-1.348$ \\
\hline
\end{tabular}

TP16, tumor suppressor protein p16; TP53, tumor suppressor protein p53; HPV, human papilloma virus; CI, confidence interval.

Table IV. Results of the univariate and multivariate analyses of selected factors for progression-free survival in all patients.

\begin{tabular}{|c|c|c|c|c|c|c|}
\hline \multirow[b]{2}{*}{ Variables } & \multicolumn{3}{|c|}{ Univariate } & \multicolumn{3}{|c|}{ Multivariate } \\
\hline & HR & $95 \% \mathrm{CI}$ & P-value & HR & $95 \% \mathrm{CI}$ & P-value \\
\hline Gender (male vs. female) & 0.624 & $0.383-1.017$ & 0.059 & & & \\
\hline Age $(<64$ vs. $\geq 64)$ & 0.929 & $0.593-1.456$ & 0.749 & & & \\
\hline Smoking (never vs. ever) & 2.012 & $1.254-3.229$ & 0.004 & 0.274 & $0.822-2.408$ & 0.213 \\
\hline $\begin{array}{l}\text { Histology (adenocarcinoma vs. squamous } \\
\text { cell carcinoma) }\end{array}$ & 2.168 & $1.273-3.695$ & 0.004 & 0.306 & $1.105-3.662$ & 0.022 \\
\hline Histological differentiation (well vs. poor) & 0.873 & $0.535-1.424$ & 0.586 & & & \\
\hline Lymph node metastasis (yes vs. no) & 2.576 & $1.493-4.443$ & 0.001 & 0.292 & $1.357-4.256$ & 0.003 \\
\hline Distant metastasis (yes vs. no) & 1.294 & $0.824-2.032$ & 0.264 & & & \\
\hline HPV (positive vs. negative) & 0.689 & $0.409-1.161$ & 0.162 & & & \\
\hline TP16 (positive vs. negative) & 0.562 & $0.341-0.924$ & 0.023 & 0.261 & $0.404-1.125$ & 0.132 \\
\hline TP53 (positive vs. negative) & 1.102 & $0.698-1.738$ & 0.678 & & & \\
\hline
\end{tabular}

HPV, human papilloma virus; CI, confidence interval; TP16, tumor suppressor protein p16; TP53, tumor suppressor protein p53; HR, hazard ratio.
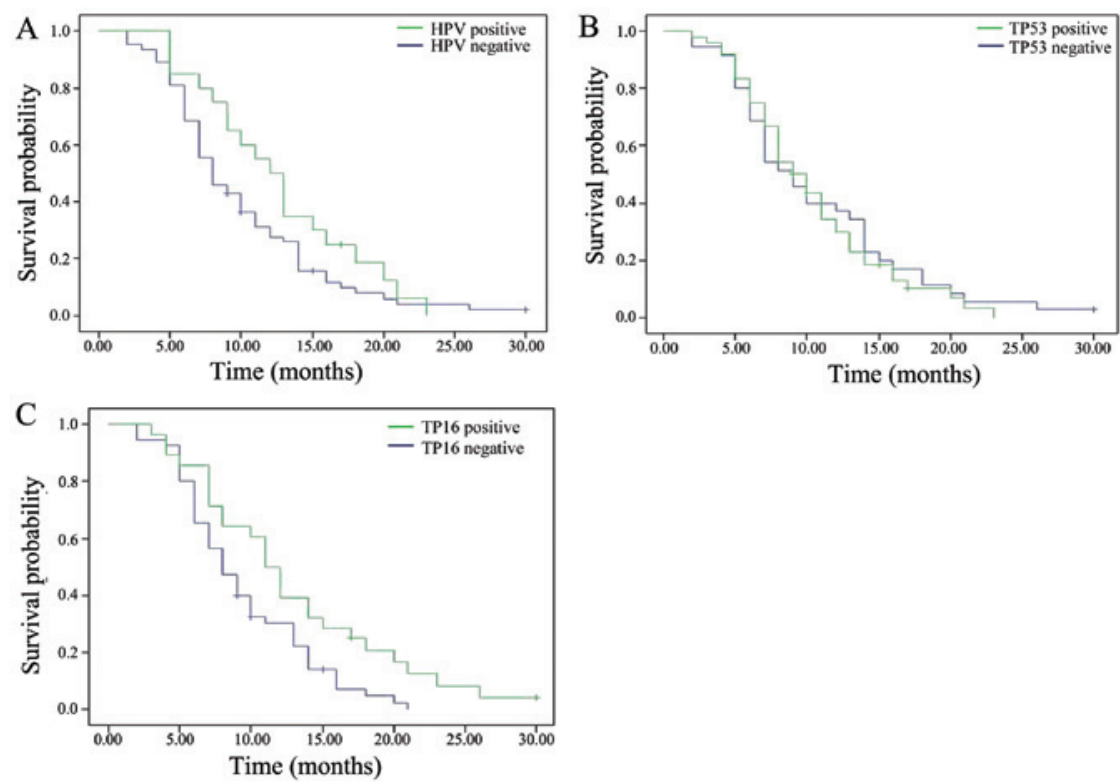

Figure 2. Kaplan-Meier plots illustrating the effects of HPV status, TP53 expression, and TP16 expression on progression-free survival (PFS) in patients with NSCLC. (A) Plots of PFS for the HPV-positive and HPV-negative populations. Median survival for HPV-positive patients (green; $\mathrm{n}=20$, of whom 1 died) was 12 months, compared with 8 months for HPV-negative patients (blue; $n=63$, of whom 4 died). (B) Plots of PFS for the TP53-positive and negative patient groups. Median survival was 9 months for both the TP53-positive (green) and TP53-negative (blue) patient groups. (C) PFS for the TP16-positive and negative patient groups. Median survival for TP16-positive patient group (green; N=28, of whom 2 died) was 11 months, compared with 8 months for TP16-negative group (blue; $\mathrm{N}=55$, of whom 3 died). 
other subgroups. However, we did not find this trend for the TP53-positive subgroups.

Although the most common cause of lung cancer is long-term exposure to tobacco smoke, $15 \%$ of men and $53 \%$ of women with lung cancer (worldwide) have cancers that are not attributable to tobacco smoking (24). The possibility of HPV involvement in bronchial squamous cell carcinoma was first suggested in 1979, and since then, several studies have provided strong support for a role for HPV as an etiological agent of lung cancer (25). A recent study found that the prevalence of HPV in NSCLC was $28.1 \%$ in Asian countries ( $\mathrm{N}=2337$ NSCLC cases), 8.4\% in European countries $(n=1553)$ and $21.3 \%$ in North and South America $(n=160)(26)$. The frequency of HPV infection in Asian NSCLC samples was higher when compared with lung cancer samples from Europe and the US. To date, $>100$ subtypes of HPV have been identified. HPV 16 and HPV 18 are the most common subtypes associated with cancer, although numerous other types (HPV 6, 11, 31, 33, 39, 52, 58, and 82) have also been detected $(27,28)$. In the present study, we detected HPV 16 , $18,33,58,6$, and 11 in patient samples, although HPV 16 was detected in the majority of cases. The overall frequency of HPV-DNA detection in NSCLC patients' samples was $24 \%$ less than the mean number of HPV cases in Asian NSCLC samples. Given that the 83 patients in our study were diagnosed with stage III-IV disease, we hypothesize that HPV infection may be associated with disease stage and metastasis in lung cancer.

Recent studies have shown that for HPV-related NSCLC, patients are more likely to be female, non-smoking, have poorly differentiated tumors, present at a late clinical stage, and exhibit more lymph node metastasis (29). In the present study, we also identified that HPV infection was significantly associated with poor differentiation, never-smokers and lymph node metastasis. Therefore, our findings are consistent with those reported by others.

The majorities of patients diagnosed with NSCLC present with advanced stage disease and have extremely poor prognoses. Currently there are no widely accepted independent prognostic factors to evaluate prognosis and survival of advanced NSCLC. Molina-Vial et al have demonstrated that nondisruptive mutations of TP53 are an independent prognostic factor of shorter survival in advanced NSCLC (30). In this study, TP53-positive patients with HPV-related NSCLC were more numerous than TP53-negative patients. Although the difference was not statistically significant, the average survival of TP53-positive patients implies that poor prognosis is associated with this clinical phenotype. However, more clinical samples are needed to confirm this hypothesis.

The present study has demonstrated that there is no significant correlation between TP16 expression and HPV-positivity in NSCLC patients. In a recent study, Gatta et al (31). Analyzed TP16 protein expression on an 83-NSCLC tissue sample microarray by immunohistochemistry and also found no association between the presence of HPV DNA and TP16 protein expression. However, our survival analysis indicates that TP16 positivity is associated with longer survival in NSCLC. Although TP16 may be a prognostic indicator in NSCLC patients, controversy regarding geographical differences as well as differences in tissue types still exist (32).
The present study provides new possibilities in the prognosis and diagnosis of advanced NSCLC. The role of TP16 in NSCLC disease progression will be investigated in subsequent studies.

In conclusion, we have demonstrated that TP53 and TP16 protein expression is not associated with the expression of HPV DNA, but that TP16 positivity may be an independent prognostic factor of longer survival in advanced NSCLC. Clinical trials are now warranted to determine whether TP16-negative patients would benefit from drugs that improve the expression of TP16.

\section{Acknowledgements}

The current study was supported by the National Natural Science Foundation of China (grant no. 81172172).

\section{References}

1. Bray F, Jemal A, Grey N, Ferlay J and Forman D: Global cancer transitions according to the Human Development Index (2008-2030): A population-based study. Lancet Oncol 13: 790-801, 2012.

2. Murray CJ and Lopez AD: Alternative projections of mortality and disability by cause 1990-2020: Global burden of disease study. Lancet 349: 1498-1504, 1997.

3. Lam WK, White NW and Chan-Yeung MM: Lung cancer epidemiology and risk factors in Asia and Africa. Int J Tuberc Lung Dis 8: 1045-1057, 2004.

4. Rezazadeh A, Laber DA, Ghim SJ, Jenson AB and Kloecker G: The role of human papilloma virus in lung cancer: A review of the evidence. Am J Med Sci 338: 64-67, 2009.

5. Castle PE, Cuzick J, Stoler MH, Wright TC Jr, Reid JL, Dockter J, Giachetti C and Getman D: Detection of human papillomavirus 16,18 , and 45 in women with ASC-US cytology and the risk of cervical precancer: Results from the CLEAR HPV study. Am J Clin Pathol 143: 160-167, 2015.

6. Slama J, Sehnal B, Dusek L, Zima T and Cibula D: Impact of risk factors on prevalance of anal HPV infection in women with simultaneous cervical lesion. Neoplasma 62: 308-314, 2015.

7. Zur Hausen H: Papillomaviruses in the causation of human cancers-a brief historical account. Virology 384: 260-265, 2009.

8. Park MS, Chang YS, Shin JH, Kim DJ, Chung KY, Shin DH, Moon JW, Kang SM, Hahn CH, Kim YS, et al: The prevalence of human papillomavirus infection in Korean non-small cell lung cancer patients. Yonsei Med J 48, 69-77, 2007.

9. Isa SI, Kurahara Y, Yamamoto S, Tamiya A, Omachi N, Asami K, Okishio K, Utsumi T, Ito N, Yoon HE, et al: Molecular analysis of human papillomavirus in never-smokers with non-small cell lung cancer. Oncol Lett 9: 927-929, 2015.

10. Ragin C, Obikoya-Malomo M, Kim S, Chen Z, Flores-Obando R, Gibbs D, Koriyama C, Aguayo F, Koshiol J, Caporaso NE, et al: HPV-associated lung cancers: An international pooled analysis. Carcinogenesis 35: 1267-1275, 2014

11. Cheng YW, Chiou HL, Sheu GT, Hsieh LL, Chen JT, Chen CY, Su JM and Lee H: The association of human papillomavirus 16/18 infection with lung cancer among nonsmoking Taiwanese women. Cancer Res 61: 2799-2803, 2001.

12. Srinivasan M, Taioli E and Ragin CC: Human papillomavirus type 16 and 18 in primary lung cancers-a meta-analysis. Carcinogenesis 30: 1722-1728, 2009.

13. Koshiol J, Rotunno M, Gillison ML, Van Doorn LJ, Chaturvedi AK, Tarantini L, Song H, Quint WG, Struijk L, Goldstein AM, et al: Assessment of human papillomavirus in lung tumor tissue. J Natl Cancer Inst 103: 501-507, 2001.

14. Proctor I, Stoeber K and Williams GH: Biomarkers in bladder cancer. Histopathology 57: 1-13, 2010.

15. Helt AM, Funk JO and Galloway DA: Inactivation of both the retinoblastoma tumor suppressor and $\mathrm{p} 21$ by the human papillomavirus type $16 \mathrm{E} 7$ oncoprotein is necessary to inhibit cell cycle arrest in human epithelial cells. J Virol 76: 10559-10568, 2002.

16. Murao K, Yoshioka R and Kubo Y: Human papillomavirus infection in Bowen disease: Negative p53 expression, not p16INK 4a overexpression, is correlated with human papillomavirus- associated Bowen disease. J Dermatol 41: 878-884, 2014. 
17. Vainshtein J, McHugh JB, Spector ME, Walline HM, Komarck CM, Stenmark MH, Prince ME, Worden FP, Wolf GT, Bradford CR, et al: Human papillomavirus-related oropharyngeal cancer: HPV and p16 status in the recurrent versus parent tumor. Head Neck 37: 8-11, 2015.

18. Maniakas A, Moubayed SP, Ayad T, Guertin L, Nguyen-Tan PF, Gologan O, Soulieres D and Christopoulos A: North-American survey on HPV-DNA and p16 testing for head and neck squamous cell carcinoma. Oral Oncol 50: 942-946, 2014.

19. Umbreit C, Aderhold C, Faber A, Sauter A, Hofheinz RD, Stern-Straeter J, Hoermann K and Schultz JD: Imatinib-associated matrix metalloproteinase suppression in p16-positive squamous cell carcinoma compared to HPV-negative HNSCC cells in vitro. Oncol Rep 32: 668-676, 2014

20. Zhang EY and Tang XD: Human papillomavirus type 16/18 oncoproteins: Potential therapeutic targets in non-smoking associated lung cancer. Asian Pac J Cancer Prev 13: 5363-5369, 2012.

21. Suzuki Y, Oonishi T, Kudo T and Doi H: LKB1, TP16, EGFR and KRAS somatic mutations in lung adenocarcinomas from a Chiba Prefecture, Japan cohort. Drug Discov Ther 6: 24-30, 2012.

22. Trbusek M, Smardova J, Malcikova J, Sebejova L, Dobes P, Svitakova M, Vranova V, Mraz M, Francova HS, Doubek M, et al: Missense mutations located in structural p53 DNA-binding motifs are associated with extremely poor survival in chronic lymphocytic leukemia. J Clin Oncol 29: 2703-2708, 2011.

23. Vainshtein J, McHugh JB, Spector ME, Walline HM, Komarck CM, Stenmark MH, Prince ME, Worden FP, Wolf GT, Bradford CR, et al: Human papillomavirus-related oropharyngeal cancer: HPV and p16 status in the recurrent versus parent tumor. Head Neck 37: 8-11, 2015.

24. Reck M, Popat S, Reinmuth N, De Ruysscher D, Kerr KM and Peters S: Metastatic non-small-cell lung cancer (NSCLC): ESMO clinical practice guidelines for diagnosis, treatment and follow-up. Ann Oncol 25 (Suppl 3): iii27-iii39, 2014.
25. Syrjanen KJ: Condylomatous changes in neoplastic bronchial epithelium. Respiration 38: 299-304, 1979.

26. Hasegawa $\mathrm{Y}$, Ando $\mathrm{M}$, Kubo A, Isa S, Yamamoto $\mathrm{S}$ Tsujino K, Kurata T, Ou SH, Takada M and Kawaguchi T: Human papilloma virus in non-small cell lung cancer in never smokers: A systematic review of the literature. Lung Cancer 83: 8-13, 2014.

27. Gillison ML, Koch WM, Capone RB, Spafford M, Westra WH, Wu L, Zahurak ML, Daniel RW, Viglione M, Symer DE, et al: Evidence for acausal association between human papillomavirus and a subset of head and neck cancers. J Natl Cancer Inst 92: 709-720, 2000.

28. Kreimer AR, Clifford GM, Boyle P and Franceschi S: Human papillomavirus types in head and neck squamous cell carcinomas worldwide: A systematic review. Cancer Epidemiol Biomarkers Prey 14: 467-475, 2005.

29. Subramanian J and Govindan R: Molecular genetics of lung cancer in people who have never smoked. Lancet Oncol 9: 676-682, 2008.

30. Molina-Vila MA, Bertran-Alamillo J, Gascó A, Mayo-de-las-Casas C, Sánchez-Ronco M, Pujantell-Pastor L, Bonanno L, Favaretto AG, Cardona AF, Vergnenègre A, et al: Nondisruptive p53 mutations are associated with shorter survival in patients with advanced non-small cell lung cancer. Clin Cancer Res 20: 4647-4659, 2014.

31. Gatta LB, Balzarini P, Tironi P, Berenzi A, Benetti A, Angiero F, Grigolato $\mathrm{P}$ and Dessy E: Human papillomavirus DNA and p16 gene in squamous cell lung carcinoma. Anticancer Res 32: 3085-3089, 2012.

32. Su CY, Chang YC, Chan YC, Lin TC, Huang MS, Yang CJ and Hsiao M: MTAP is an independent prognosis marker and the concordant loss of MTAP and p16 expression predicts short survival in non-small cell lung cancer patients. Eur J Surg Oncol 40: 1143-1150, 2014. 